\title{
Influenza Pandemics and the Associated Bacterial Infections
}

\author{
Khanna $\mathbf{M}^{1 *}$, Agrawal $\mathbf{N}^{1,2}$, Chandra $\mathbf{R}^{3,4}$ and \\ Dhawan $\mathbf{G}^{2 *}$ \\ ${ }^{1}$ Department of Virology (A Unit of Department of \\ Microbiology), Vallabhbhai Patel Chest Institute, \\ University of Delhi, Delhi, India \\ ${ }^{2}$ Department of Biomedical Science, Acharya Narendra \\ Dev College, University of Delhi, Kalkaji, New Delhi, \\ India \\ ${ }^{3}$ Dr. B.R Ambedkar Center for Biomedical Research, \\ University of Delhi, Delhi, India \\ ${ }^{4}$ Department of Chemistry, University of Delhi, Delhi, \\ India \\ *Corresponding author: Madhu Khanna, Department \\ of Virology (A Unit of Dept. of Microbiology), Vallabhbhai \\ Patel Chest Institute, University of Delhi, Delhi-110007, \\ India
}

Gagan Dhawan, Department of Biomedical Science, Acharya Narendra Dev College, University of Delhi, Kalkaji, New Delhi-110019, India

Received: August 10, 2017; Accepted: September 15, 2017; Published: September 25, 2017

\section{Introduction}

Influenza A virus is major respiratory pathogen responsible for causing highly contagious and acute respiratory disease. It belongs to the family of RNA viruses "Orthomyxoviridae" and has a $13.5 \mathrm{~kb}$ genome with eight single-stranded (ss) RNA segments. These negative-sense ssRNA segments encode eleven proteins: HA, PB1, PB2, PA, NP, NEP, M1, NA, NS1, M2 and PB1-F2. HA protein facilitates entry of virus in the host cell, polymerase subunits PB1, PB2, PA and NP (nucleoprotein) assist in replication and transcription of viral RNAs. Nuclear export protein (NEP/NS2) and matrix protein (M1) plays a role in export of viral nucleoprotein from the nucleus to cytoplasm and their assembly into virion at plasma membrane. The NA protein assist in the release of virus from infected cells and NS1 protein acts as interferon antagonist inhibiting the host immune response. M2 protein is an integral part of viral envelope, forming $\mathrm{pH}$ regulated and highly sensitive proton conducting channels, essential for viral replication. PB1-F2 protein is an important determinant of virulence of influenza virus, increases the severity of secondary bacterial infections and also induces apoptosis [1].

In Influenza A virus, aquatic birds act as natural reservoir but it has the ability to infect variety of hosts like birds, human beings and swine [3]. Due to segmented nature of the genome, influenza A virus has high variability thereby undergoing re-assortment when a cell is infected with more than one virus [4]. This process of genetic reassortment results in generation of novel strains of influenza virus thus preventing the acquired immune response from previous infections, leading to recurrent epidemics and global pandemics.

Influenza virus pandemics have been defined as global outbreaks of the disease due to emergence of viruses with new antigenic subtypes. There have been four pandemics: the 1918 Spanish influenza, the 1957 Asian influenza, the 1968 Hong Kong influenza and the 2009 Swine influenza, resulting in more than a million deaths [5]. Between these episodes of pandemics, there have been various epidemics of grave severity. Influenza pandemics and epidemics are initiated by the introduction and successful adaptation of antigenic variation in the surface glycoproteins, Hemagglutinin (HA) and Neuraminidase (NA) assisting the virus in evading the host immune response [6,7]. On the basis of sequence analysis, a total of sixteen HA (H1-H16) and eleven NA (N1-N11) have been identified, combination of which results in major outbreaks [3]. The variation in viral genome occurs either as a result of minor antigenic changes over a period of time, facilitating escape from the existing immune response, known as "antigenic drift", producing outbreaks of seasonal flu or by sudden major change in the genome as a result of genetic re-assortment where the genomes of two different strains of viruses are re-assorted creating a novel viral strain, process known as "genetic shift" [8]. The outbreaks of seasonal influenza are the result of frequent anti-g-genic drift, however in case of genetic shift, if the novel strain has virulence for human; it may give rise to pandemic situation, since humans are unlikely to generate appreciable immune response against the new virus.

Earlier FDA had approved the drugs Amantidine and Rimantidine (M2 proton-selective ion channel protein inhibitors), but these drugs are abandoned for treatment owing to the high resistance ( $>99 \%)$ of Influenza A (H3N2, H1N1'09) virus for these drugs (CDC) Existing influenza treatment is limited to neuraminidase inhibitors and increasing number of drug resistance cases against these inhibitors has been reported which is serious matter of concern [9]. The most efficient treatment for influenza virus infection is through vaccination, thereby reducing the impact of pandemic influenza [10]. The currently approved vaccine provides an effective countermeasure against influenza virus, but they provide humoral immunity against the surface antigen, which often undergoes antigenic drift. Hence, these vaccines need to be reformulated annually in order to generate immune response against the specific strain of virus that is predicted to circulate in the next season, which is a major limitation [11].

\section{Bacterial Infections}

Influenza usually does not advance to death in healthy children and adults, however serious sequelae can occur with secondary or co-infection with bacterial pathogens, especially in immunecompromised individuals with chronic health conditions like respiratory ailment, cardiac disease etc. Bacterial associated pneumonia is the most common source of increased mortality during the pandemic season. Bacterial pathogens may infect concurrently with the viral infection, the co-infection results in pneumonia thereby 
increasing the severity of disease. Bacterial infection may also occur after the influenza virus has been cleared from lungs, and the host is more susceptible to secondary infections [12]. Secondary infections are facilitated by influenza-associated impairment of immune system, caused by enhanced release of inflammatory cytokines or by decreasing the ability to clear bacterial infections.

Clinicians now have several ways to alleviate pneumonia through vaccines, antibiotics and antiviral therapies, thereby contributing to decreasing the burden of disease globally. It has been observed that influenza and pneumococcal vaccine in synergy, reduced hospitalization due to influenza and pneumonia significantly [13]. In the cases of suspected invasive bacterial infection, early initiation of antiviral therapy and appropriate antibiotics should be administered to increase the efficiency of the treatment.

These measures however have limitations, which restrain their effectiveness. The over usage of antibiotics to combat bacterial infections, has contributed severely to the antibiotic resistance with evidence that MRSA (Methicillin-resistant Staphylococcus aureus) infections is responsible for increase in morbidity and mortality, especially among the children diagnosed with influenza [14]. Limitations include the delay in production of vaccines and stockpiling of antiviral and antibacterial drugs [15].

\section{Pandemics}

\section{H1N1 Pandemic}

The 1918 Pandemic "Spanish flu" remains unprecedented in terms of severity, killing about 50-100 million people globally, hence often known as "mother of all pandemics" $[16,17]$. The causative organism was the H1N1 subtype of Influenza A virus with avian ancestral source. It was highly intriguing how the viruses of avian origin adapt to mammalian hosts and infect such different cell types. The examination of genome revealed the possibility of de novo adaptation of the avian virus by parallel evolution of genes in a novel (human) host [18]. The pandemic is believed to have originated from china and occurred in three waves, starting from a mild wave in spring season, followed by the most catastrophic and severe wave in fall and then the final mild wave in winter of 1918-1919 [19]. Pandemic reached Indian subcontinent through Bombay, thereby spreading North and south simultaneously, increasing the death toll to 10-20 million (38\% of global mortality), making India the worst affected country in terms of mortality $[16,19,20]$.

Although there were various theories regarding the severity of 1918 pandemic, the experts reached a consensus that the high mortality rate was due to secondary infections caused by bacterial pathogens (pneumococci, streptococci, staphylococci) colonizing the upper respiratory tract [21]. Experts believed that bacterial invaders infected in sequential manner, after the influenza virus cripple the pulmonary tissue [22]. Most commonly identified bacteria in the pandemic patients were S. pneumoniae, S. pyogenes and less commonly S. aureus and H. influenzae [23]. One of the most puzzling features of 1918 Pandemic was the W-shaped Influenza mortality curve with unusual burden among the young adults (healthy population between 20-40 years), instead of the usual U-shaped curve $[6,18,24]$.Various reports have shown that the increased mortality in otherwise healthy young could be contributed by the excessive release of pro-inflammatory cytokines (IL-6, IL-8) and tumor necrosis factor $(\mathrm{TNF}-\alpha)$ [25-27].

Another reason for the devastation by 1918 pandemic could be the rudimentary health practices with limited knowledge about disease prevention and control. The development of antivirals, vaccines and antibiotics to treat the secondary infections were still decades away, hence efforts to control the outbreaks were restricted to NonPharmaceutical Interventions (NPIs), which included quarantine, prohibition of public gatherings and use of facemasks [28].

Spanish flu is still believed to be the worst public disaster in the history, killing millions of people. However, it brought to light the urgency to improve the public health care conditions across the globe, which led to major advancements in medical sciences, awareness and better preparedness for such un-anticipated outbreaks.

\section{H2N2 Pandemic}

After almost 40 years of Spanish flu, a novel strain of Influenza virus (H2N2) of avian origin, emerged in China in February 1957, and gave rise to a pandemic situation, killing around 500,000 to 2 million people worldwide [29]. After spreading across China, the Asian flu progressed to Singapore, Japan and Taiwan before traversing across the globe. The H2N2 strain was the product of re-assortment between the circulating human virus that introduced $\mathrm{N} 2$ and avian virus with $\mathrm{H} 2 \mathrm{HA}$, as revealed from phylogenetic studies $[1,30]$. As with Spanish Flu, H2N2 virus would reappear in successive waves, second one being more severe than the previous one [31]. Asian flu reached India in May 1957 through the port of Madras, thereafter spreading throughout the country, leading to the death of about 1098 people from May 1957- February 1958 [32].

The Asian influenza had similar characteristics of increased deaths due to bacterial pneumonia with $S$. aureus, $H$. influenza and $S$. pneumoniae being the major pathogens that were isolated $[21,22]$. The Influenza mortality curve shifted towards younger age group, similar to 1918 Pandemic, suggesting the presence of pre-existing antibodies in elderly from the prior exposure [33]. By the time of Asian flu, global surveillance was used to determine the disease burden through a network of laboratories worldwide, linked to Influenza Research Center based in London [34]. After the catastrophic effect of Spanish flu, several measures were taken in the field of influenza research to be better prepared for such unforeseen situations, although the expertise was still inadequate. The 1957 pandemic was the first event to study the response of vaccination in large population that has not been exposed to the novel $\mathrm{H}_{2} \mathrm{~N}_{2}$ strain of virus, but did not have a significant impact due to limited supply [35]. Antibiotics reduced the disease burden due to secondary bacterial infection; however they were not effective against viral infections [34]. The usage of nonpharmaceutical interventions was minimum and the antivirals were yet to be developed [36]. Asian flu, though mild pandemic, emerged as a reminder of persisting global threat of Influenza virus.

\section{H3N2 Pandemic}

A decade after its emergence, Asian flu underwent genetic reassortment between human and avian strain via antigenic shift, giving rise to a novel H3N2 strain and triggering a new pandemic situation known as Hong Kong flu. Even though this strain of virus was highly contagious leading to rapid dissemination globally, it was still milder 
than Asian flu with the mortality estimates of 500,000 to 2 million deaths worldwide [29]. After being first reported in Hong Kong in July 1968, it spread throughout Asia before reaching west coast of United States in August, England and Australia by September, Canada in December and France by January 1969 [30,33]. The H3N2 virus reached Madras, India in September from Singapore followed by the reported decline in influenza activity during end of November and December in Madras. It gradually spread to entire Indian subcontinent with the appearance of most severe manifestation among children [37].

A characteristic shift in mortality curve was observed with highest fatality cases being reported among the children and elderly, forming a U-shaped mortality curve [38]. Similar to previous pandemics, it spread in two successive waves but the distinctive feature of this pandemic was that the number of associated deaths in the two waves varied with geographic location, with United states and Canada being more affected by the first wave, whereas Europe and Asia by the second wave, thereby following a smoldering pattern [33]. The relative amelioration of infection rates can be the consequence of the pre-existing antibodies to neuraminidase antigen (N2), similar to its antecedent Asian flu (H2N2) strain.

The foremost complication during the Hong Kong pandemic was pneumonia (associated with Influenza and staphylococcus), but due to advances in the field of antibacterial therapies, the mortality rate was higher from primary influenza associated pneumonia rather than in synergy with secondary bacterial infections [22,38]. Similar to the other infective parts of the world, in India the pandemic was relatively mild with few complications like pneumonia, bronchopneumonia, streptococci and staphylococcus isolation from sputum, gastrointestinal symptoms etc. [37]. Due to less severity and low mortality rates, the control measures ascertain the use of vaccines and antibiotics in the case of secondary bacterial infections (pneumonia), rather than more costly non-pharmaceutical interventions [39]. The vaccines were developed against the circulating virus but were made available only when the pandemic had peaked indicating towards the lack of progress in healthcare strategies from 1957 Asian flu pandemic [40].

\section{H1N1 Pandemic}

The H1N1/09 virus commonly known as swine flu, emerged in April 2009 with Mexico being the epicenter and was declared as the first global pandemic of 21st century on 11 June 2009 by WHO $[3,6,8]$. Swine flu is believed to be the fourth generation descendant of Swine flu that was first described in 1918 and emerged from the triple re-assortment between human, swine and avian influenza A virus to form the H1N1/09 pandemic strain [3,6,31]. After the pandemic declaration, national pandemic preparedness plans were put in motion globally, which included the use of antiviral therapy, disease alleviation and treatment [41]. The virus spread at unprecedented speed across the world with the mortality estimates of 575,000. Similar to the previous pandemics of $20^{\text {th }}$ century, the swine flu exhibited the wave pattern of dissemination, which varied geographically. For example in North America, the pandemic had a two-wave behavior with the peaks being observed during spring-summer and fall [42]. In India however three wave patterns was observed, with peaks during September 2009, December 2009 and August 2010 [43]. The index cases in India were identified from Pune, which soon spread to the entire nation [44].

The characteristics of Influenza H1N1/09 were similar to the seasonal influenza, infected individuals became more prone to underlying conditions, which further exacerbated the infection and increased the number of cases requiring hospitalization [45]. Complications seen in the patients included bacterial and viral pneumonia, asthma, lung and heart disease etc. Pneumonia caused by secondarybacterial infections and acute respiratory distress syndromes were the major cause of serious complications and mortality during 1918 Spanish flu [21,46]. Bacterial co-infections also played a major role in fatal cases of $\mathrm{H} 1 \mathrm{~N} 1 / 09$ pandemic with the S. pneumonia being most prevalent, followed by S. pyogenes, S. aureus (MRSA), S. mitis, H. influenzae being isolated from lung specimen of fatal cases [47]. Similarly in India, the severity of pandemic was associated mainly by secondary infections, like primary viral pneumonia and secondary bacterial pneumonia along with exacerbation of other chronic health conditions [48]. Apart from secondary bacterial infections, there were reports of viral co-infection leading to further exacerbation of the disease. The respiratory viruses like RSV, rhino virus, corona virus, metapneumovirus, parainfluenza co-infected the pandemic H1N1 cases, increasing the severity of the disease [22]. There was a shift in mortality curve, with the younger populations (children, young adults and pregnant women), being worst affected because the elderly are more likely to contain neutralizing antibodies from previous exposure to $\mathrm{H} 1 \mathrm{~N} 1$ virus $[49,50]$.

Since its emergence, H1N1/09 virus was more susceptible to antivirals that were neuraminidase inhibitors (oseltamivir, zanamir) and resistant to adamantanes (amantadines, rimantadines). The antivirals were found to be most effective in patients with severe influenza illness and reducing secondary bacterial infections, when started within 48 hours of the onset of symptoms [22,50]. In the area of limited antiviral availability, the decision to start the antiviral therapy was based clinicians judgment, as the patients with mild symptoms did not require the antivirals unless they are at the risk of associated complications [46]. Clinician also prescribed antibacterial drugs in case bacterial co-infection was suspected, taking into account the data regarding the frequency of pathogen isolated during the cases of coinfection [3]. The alternative mode of treatment was vaccines, which were developed within 6 months and were the best tools to prevent the unforeseen spread of pandemic. Two types of vaccines were developed which were approved by FDA, adjuvant and non-adjuvant, both of which were safe and immunogenic, hence used widely during 2009 Pandemic situation.

The overall response to 2009 pandemic situation displayed a significant improvement in the preparedness plans by better surveillance schemes to ensure rapid detection and response to pandemics [50]. In comparison to previous pandemics, the pandemic of the $21^{\text {st }}$ century was dealt with combined use of vaccines and antivirals, which undoubtedly reduced the morbidity and mortality. The non-pharmaceutical interventions like hand hygiene, isolation of symptomatic individuals, played an important role in containment of influenza pandemic [51].

Overall, the 2009 pandemic were mild but it caused a major socioeconomic burden, which was more comprehensively documented than previous pandemics of last century. Though it reinforced 
optimism about better preparedness, but the cost-effectiveness of the healthcare facilities were still a matter of concern.

\section{Conclusion}

Influenza pandemics are one of the major threats to the world because of their high morbidity and mortality. The influenza related mortalities are mostly not due to primary viral infection but due to secondary viral and bacterial pneumonia. Hence, strategy for prevention of future pandemics should give emphasis on the control of both bacterial and viral associated community acquired pneumonia. Another measure for better preparedness could be easy accessibility to antivirals, antibiotics and vaccines, hence priority should be given to better infrastructure facilities for rapid production of vaccines, stockpiling of antivirals and antibiotics. In addition to this, better sanitation and improved nutritional status of the society will go a long way in controlling the disease. The mortality surveillance plans would be helpful for better understanding of disease burden of influenza, the pathogens contributing to the mortality and the most vulnerable age group. It shall be helpful in designing more specific preventive strategies and thereby reducing the catastrophic effects of influenza.

\section{References}

1. Neumann G, Noda T, Kawaoka Y. Emergence and pandemic potential of swine-origin H1N1 influenza virus. Nature. 2009; 459: 931-939.

2. Chang LY, Shih SR, Shao PL, Huang DT, Huang LM. Novel swine-origin influenza virus $A(\mathrm{H} 1 \mathrm{~N} 1)$ : the first pandemic of the $21^{\text {st }}$ century. J Formos Med Assoc. 2009; 108: 526-532.

3. Hajjar SA, Mclntosh K. The first influenza pandemic of the $21^{\text {st }}$ century. Ann Saudi Med. 2010; 30: 1-10.

4. Brockwell-Staats C, Webster RG, Webby RJ. Diversity of influenza viruses in swine and the emergence of a novel human pandemic influenza A (H1N1). Influenza Other Respir Viruses. 2009; 3: 207-213.

5. Saunders-Hastings PR, Krewski D. Reviewing the history of pandemic influenza: Understanding patterns of emergence and transmission. Pathogens. 2016; 5: 66.

6. Khanna M, Saxena L, Gupta A, Kumar B, Rajput R. Influenza pandemics of 1918 and 2009. Future Virology. 2013; 8: 335-342.

7. Khanna M, Kumar P, Choudhary K, Kumar B, Vijayan VK. Emerging influenza virus: a global threat. J Biosci. 2008; 33: 475-482.

8. Khanna M, Kumar B, Gupta A, Kumar P. Pandemic Influenza A H1N1 (2009) Virus: Lessons from the past and implications for the future. Indian $\mathrm{J}$ Virol. 2012; 23: 12-17.

9. Hurt AC, Holien JK, Parker MW, Barr IG. Oseltamivir resistance and the $\mathrm{H} 274 \mathrm{Y}$ neuraminidase mutation in seasonal, pandemic and highly pathogenic influenza viruses. Drugs. 2009; 69: 2523-2531.

10. Khanna M, Sharma S, Kumar B, Rajput R. Protective immunity based on the conserved hemagglutinin stalk domain and its prospects for universal influenza vaccine development. BioMed Research International. 2014; 2014: Article ID 546274.

11. Pica N, Palese P. Toward a universal influenza virus vaccine: Prospects and challenges. Annu. Rev. Med. 2013; 64: 189-202.

12. Small CL, Shaler CR, McCormick S, Jeyanathan M, Damjanovic D, Brown EG, et al. Influenza infection leads to increased susceptibility to subsequent bacterial superinfection by impairing NK cell responses in the lung. J Immunol. 2010; 184: 2048-2056.

13. Hedlund J, Christenson B, Lundbergh P, Ortqvist A. Effects of a largescale intervention with influenza and 23-valent pneumococcal vaccines in elderly people: a 1-year follow-up. Vaccine. 2003; 21: 3906-3911.

14. Finelli L, Fiore A, Dhara R, Brammer L, Shay DK, Kamimoto L, et al.
Influenza-associated pediatric mortality in the United States: increase of staphylococcus aureus coinfection. Pediatrics. 2008; 122: 805-811.

15. Cinti SK, Barnosky AR, Gay SE, Goold SD, Lozon MM, Kim K, et al. Bacterial pneumonias during an influenza pandemic: how will we allocate antibiotics? Biosecur Bioterror. 2009; 7: 311-316.

16. Johnson NP, Mueller J. Updating the accounts: global mortality of the 19181920 "Spanish" influenza pandemic. Bull Hist Med. 2002; 76: 105-115.

17. Khanna M, Saxena L, Gupta A, Kumar B, Rajput R. Influenza pandemics of 1918 and 2009. Future Virology. 2013; 8: 335-342.

18. Taubenberger JK, Morens DM. Influenza: the mother of all pandemics. Emerg Infect Dis. 2006; 12: 15-22.

19. Chandra $S$ and Kassens-Noor E. The evolution of pandemic influenza: evidence from India, 1918-1919. BMC Infect Dis. 2014; 14: 510.

20. Chandra S, Kuljanin G, Wray J. Mortality from the influenza pandemic of 1918-1919: The case of India. Demography. 2012; 49: 857-865.

21. Morens DM, Taubenberger JK, Fauci AS. Predominant role of bacterial pneumonia as a cause of death in pandemic influenza: Implications for pandemic influenza preparedness. J Infect Dis. 2008; 198: 962-970.

22. Joseph C, Togawa Y, Shindo N. Bacterial and viral infections associated with influenza. Influenza and Other Respiratory Viruses. 2013; 7: 105-113.

23. Morens DM, Taubenberger JK, Harvey HA, Memoli MJ. The 1918 influenza pandemic: Lessons for 2009 and the future. Crit Care Med. 2010; 38: e10-e20.

24. Luk J, Gross P, Thompson WW. Observations on mortality during the 1918 influenza pandemic. Clin Infect Dis. 2001; 33: 1375-1378.

25. Osterholm MT. Preparing for the next pandemic. N Engl J Med. 2005; 352: 1839-1842.

26. Kash JC, Basler CF, García-Sastre A, Carter V, Billharz R, Swayne DE, et al. Global host immune response: pathogenesis and transcriptional profiling of type A influenza viruses expressing the hemagglutinin and neuraminidase genes from the 1918 pandemic virus. J Virol. 2004; 78: 9499-9511.

27. Kobasa D, Takada A, Shinya K, Hatta M, Halfmann $P$, Theriault $S$, et al. Enhanced virulence of influenza $A$ viruses with the haemagglutinin of the 1918 pandemic virus. Nature. 2004; 431: 703-707.

28. Markel H, Stern AM, Navarro JA, Michalsen JR, Monto AS, DiGiovanni C. Nonpharmaceutical influenza mitigation strategies, US communities, 19181920 pandemic. Emerg. Infect. Dis. 2006; 12: 1961-1964.

29. Guan Y, Yikaykrishna D, Bahl J, Zhu H, Wang J, Smith GJ. The emergence of pandemic influenza viruses. Protein Cell. 2010; 1: 9-13.

30. Kilbourne ED. Influenza pandemics of the $20^{\text {th }}$ century. Emerg Infect Dis. 2006; 12: 9-14

31. Scalera NM, Mossad SB. The first pandemic of the $21^{\text {st }}$ century: Review of the 2009 pandemic variant influenza A (H1N1) virus. Postgrad Med. 2009; 121: 43-47.

32. Menon IGK. The 1957 pandemic of influenza in India. Bull World Health Organ. 1959; 20: 199-224.

33. Viboud C, Grais R, Lafont B, Miller M, Simonsen L. Multinational impact of Hong Kong influenza pandemic: Evidence for a smoldering pandemic. J. Infect. Dis. 2005; 192: 233-249.

34. Jackson C. History lessons: The Asian flu pandemic. Br. J. Gen. Pract. 2009; 59: 622-623.

35. Henderson D, Courtney B, Inglesby T, Toner E, Nuzzo J. Public health and medical responses to the 1957-58 influenza pandemic. Biosecur. Bioterror. 2009; 7: 265-273.

36. Trotter Y, Dunn FL, Drachmann RH. Asian influenza in the United States. Am. J. Hyg. 1959; 70: 34-50.

37. Veeraraghavan N. Hong Kong Influenza in Madras State, India, 1968. Bull World Health Organ. 1969; 41: 399-400.

38. Hsieh YC, Wu TZ, Liu DP, Shao PL, Chang LY, Lu CY, et al. Influenza 
pandemics: Past, present and future. J Formos Med Assoc. 2006; 105: 1-6.

39. Taubenberger JK, Morens DM. Influenza: The once and future pandemic Public Health Rep. 2010; 125: 16-26.

40. Rogers K. Hong Kong Flu of 1968.

41. Berera D, Zambon M. Antivirals in the 2009 pandemic - lessons and implications for future strategies. Influenza Other Respir Viruses. 2013; 7: 72-79.

42. Cook S, Conrad C, Fowlkes AL, Mohebbi MH. Assessing google flu trends performance in the United States during the 2009 influenza virus a (H1N1) pandemic. PLoS ONE. 2011; 6: e23610.

43. Mishra B. 2015 resurgence of influenza A (H1N1) 09: Smoldering pandemic in India? J. Glob. Infect. Dis. 2015; 7: 56-59.

44. Mehta AA, Kumar VA, Nair SG, Joseph FK, Kumar G, Singh SK. Clinical profile of patients admitted with swine-origin influenza $A(H 1 N 1)$ virus infection: An experience from A tertiary care hospital. J Clin Diagn Res. 2013; 7: 2227-2230.

45. CDC. Swine-origin influenza A (H1N1) virus infection in a school-New York City, April 2009. MMWR. 2009(a); 58: 470-472.

46. Sinha M. Swine flu. Journal of Infection and Public Health. 2009; 2: 157-166.

47. CDC. Bacterial coinfections in lung tissue specimen from fatal cases of 2009 pandemic influenza A (H1N1)-United States. MMWR. 2009(b); 58: 1-4
48. Dandagi GL, Byahatti SM. An insight into the swine-influenza $A(H 1 N 1)$ virus infection in humans. Lung India. 2011; 28: 34-38.

49. Girard MP, Tam JS, Assossou OM, Kieny MP. The 2009 A (H1N1) influenza virus pandmeic: A review. Vaccine. 2010; 31: 4895-4902.

50. del Rio C, Guarner J. The 2009 influenza A (H1N1) pandemic: what have we learned in the past 6 months. Trans Am ClinClimatol Assoc. 2010; 121 128-137.

51. Cantey PT, Chuk MG, Kohl KS, Herrmann J, Weiss P, Graffunder CM, et al. Public health emergency preparedness: lessons learned about monitoring of interventions from the national association of county and city health official's survey of nonpharmaceutical interventions for pandemic H1N1. J Public Health Manag Pract. 2013; 19: 70-76.

52. CDC. Antiviral drug resistance.

53. Khanna M, Gupta N, Gupta A, Vijayan VK. Influenza A (H1N1) 2009: A pandemic alarm. Journal of Biosciences. 2009(a); 34: 481-489.

54. Khanna M, Kumar B, Gupta N, Kumar P, Gupta A, Vijayan VK, et al. Pandemic swine influenza virus (H1N1) A threatening evolution. Indian J Microbiol. 2009(b); 49: 365-369. 